03,10

\title{
Эволюция симметрии промежуточных фаз и их фононных спектров в процессе топохимического превращения кремния в карбид кремния
}

\author{
(C) Ю.Э. Китаев ${ }^{1,2}$, С.А. Кукушкин ${ }^{2-4, \uparrow}$, А.В. Осипов ${ }^{2,3}$ \\ ${ }^{1}$ Физико-технический институт им. А.Ф. Иофрее РАН, \\ Санкт-Петербург, Россия \\ ${ }^{2}$ Институт проблем машиноведения РАН, \\ Санкт-Петербург, Россия \\ ${ }^{3}$ Санкт-Петербургский национальный исследовательский университет \\ информационных технологий, механики и оптики, \\ Санкт-Петербург, Россия \\ ${ }^{4}$ Санкт-Петербургский государственный политехнический университет Петра Великого, \\ Санкт-Петербург, Россия \\ ฯ E-mail: sergey.a.kukushkin@gmail.com
}

(Поступила в Редакцию 29 июня 2016 г.)

\begin{abstract}
Выполнен симметрийный анализ кристаллической структуры и фононного спектра в процессе непрерывного превращения кремния в карбид кремния. Определена трансформация симметрии фононов в точках высокой симметрии зоны Бриллюэна при переходе от исходной кубической структуры кремния (алмаза) через промежуточную кубическую структуру $\mathrm{SiC}$ к тригональной структуре $\mathrm{SiC}$. Установлены правила отбора для ИК и рамановских спектров во всех трех фазах.
\end{abstract}

Авторы благодарят за финансовую поддержку РФФИ (гранты № 15-0306155 и 16-29-03149-офи-м).

DOI: 10.21883/FTT.2017.01.43946.267

Современные технологические методы роста [1-3] позволяют изготовить искусственные монокристаллические наноструктуры на основе известных полупроводников. К их числу относятся короткопериодные сверхрешетки на основе кристаллов типа цинковой обманки (например, $\left.(\mathrm{GaAs})_{m}(\mathrm{AlAs})_{n}\right)$ [4-6], вюрцита $(\mathrm{GaN})_{m}(\mathrm{AlN})_{n}[7,8]$ и алмаза $\mathrm{Si}_{m} \mathrm{Ge}_{n}[9,10]$. Кремний является одним из наиболее широко используемых материалов в качестве исходного кристалла. Замещая периодическим образом атомы кремния в исходной решетке атомами других элементов IV группы, можно получить разнообразный набор кристаллических структур, отличающихся как пространственной группой симметрии, так и размещением образующих эти искусственные кристаллы атомов. Чтобы не искать возможные структуры простым подбором, можно использовать программы Кристаллографического сервера Бильбао (BCS) [11-13], которые позволяют последовательно получить все возможные производные низкосимметричные кристаллические типы, исходя из структуры кристалла кремния.

B настоящей работе программы BCS будут использованы для анализа кристаллических структур, образующихся при превращении кремния $(\mathrm{Si})$ в карбид кремния $(\mathrm{SiC})$ при протекании химической реакции

$$
2 \mathrm{Si}(\text { solid })+\mathrm{CO}(\text { gas })=\mathrm{SiC}(\text { solid })+\mathrm{SiO}(\text { gas }) \text {. }
$$

Метод получения $\mathrm{SiC}$ по реакции (1) был теоретически предсказан и экспериментально подтвержден в цикле работ, обобщенных в обзорах $[14,15]$. В этих работах было обнаружено, что реакция (1) протекает в несколько стадий. На первой стадии один из атомов $\mathrm{Si}$, взаимодействуя с молекулой $\mathrm{CO}$, превращается в газообразную молекулу $\mathrm{SiO}$ (температура синтеза $T>950^{\circ} \mathrm{C}$ ), на его месте образуется кремниевая вакансия $\left(V_{\mathrm{Si}}\right)$, а атом углерода „затягивается“ в межузельное пространство Si. Анализ, проведенный в $[14,15]$, показал, что между углеродом в межузельном пространстве $(\mathrm{C})$ и кремниевой вакансией возникает сильное механическое упругое взаимодействие. В том случае, если они расположены вдоль направления [111] кремния, они притягиваются друг к другу, образуя устойчивый комплекс, названный в $[14,15]$ дилатационным диполем. При этом практически вся дилатационная упругая энергия, возникшая из-за внедрения атома углерода и образования вакансии, релаксирует. Скорость данной химической реакции максимальна вдоль направления, вдоль которого дилатационные диполи притягиваются, т. е. вдоль направления [111] подложки Si. На первой стадии реакции в приповерхностной области формируется ансамбль дилатационных диполей, образующих сверхрешетку. При этом образуется промежуточное метастабильное вещество, состоящее из решетки кремния и решетки диполей $\left(\mathrm{C}-V_{\mathrm{Si}}\right)$. На второй стадии химической реакции атомы $\mathrm{C}$ смещаются из своих позиций по направлению к кремниевой вакансии. При этом происходит фазовый переход первого рода типа смещения с образованием слоя $\mathrm{SiC}$. Так как объем элементарной кристаллической ячейки $\mathrm{SiC}$ значительно меньше объема элементарной ячейки $\mathrm{Si}$, возникающие 


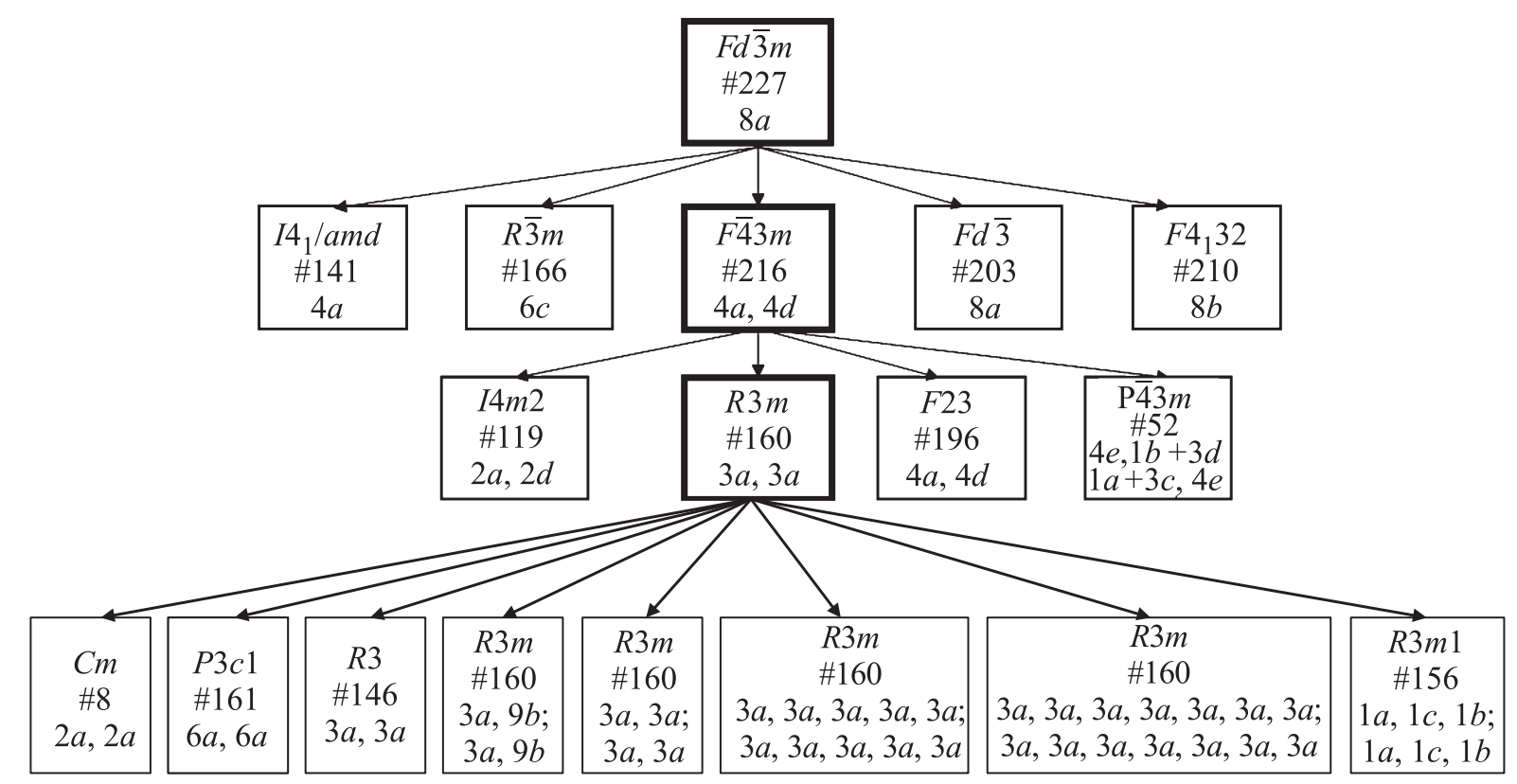

Рис. 1. Дерево соотношений группа-подгруппа для группы симметрии кремния (алмаза) $F d \overline{3} m$, выбранной в качестве аристотипа. Жирными линиями выделены структуры, рассматриваемые в настоящей работе.

в результате превращения деформации разрывают часть материала Si. При этом образуются поры под поверхностью пленки $\mathrm{SiC}$. Поскольку ансамбль диполей образует дополнительную решетку в $\mathrm{Si}$, а смещение атомов $\mathrm{C}$ по направлению к вакансии $V_{\mathrm{Si}}$ происходит строго упорядоченно вдоль направления [111], в результате образуется монокристаллическая упорядоченная пленка $\mathrm{SiC}$.

В работе [16] было показано, что образование промежуточных состояний представляет собой выгодный с точки зрения фазового перехода первого рода процесс. Образование промежуточных метастабильных веществ позволяет системе обойти высокий активационный барьер, связанный с возникновением высокой упругой энергии при прямом переходе из $\mathrm{Si}$ в $\mathrm{SiC}$. Для фазового перехода с участием химической реакции кремний-карбид кремния таким промежуточным состоянием является „предкарбидное“ состояние кремния, насыщенное дилатационными диполями, т. е. парами атом углерода-кремниевая вакансия, сильно притягивающимися друг к другу. В процессе такого перехода существенным образом меняется симметрия фаз. Карбид кремния при одном и том же химическом составе имеет более сотни различных политипов. В связи с этим весьма интересным представляется исследовать симметрию возможных фаз как промежуточных короткоживущих, так и образующихся в конечном состоянии, используя аппарат теоретико-группового анализа. Данный анализ позволяет не только определить симметрию образующихся веществ, но также определить симметрию фононных спектров.

C помощью программы MAXSUB [12] мы последовательно анализируем структурные типы, имеющие сим- метрию одной из максимальных подгрупп по отношению к исходной группе симметрии кремния. Аналогичная процедура была применена для моделирования полупроводников типа вюрцита с магнитными примесями [17]. В тех структурах с симметрией максимальной подгруппы, в которых имеется расщепление заполненных атомами кремния позиций Уайкофа, в расщепленных позициях можно разместить атомы различных химических элементов. Те подгруппы, в которых позиции симметрии исходной группы не расщепляется, соответствуют либо деформации решетки, либо являются подгруппами с нехарактеристическими орбитами [18]. Результаты анализа представлены на рис. 1. (Кратность позиций в Международных таблицах [18] и на сайте BCS [11-13] дана для кристаллографической, а не примитивной элементарной ячейки). Полученное дерево соотношений группа-подгруппа носит название дерева Бернигхаузена и процедура его построения была предложена в работах $[19,20]$ и использовалась в работе [21].

Видно, что группа симметрии алмаза (кремния) $F d \overline{3} m$ имеет пять максимальных подгрупп. В одной из них $F d \overline{3}$ заполненная позиция симметрии $8 a$ является нехарактеристической орбитой [18]. Поэтому структура с этой группой симметрии $F d \overline{3}$, в которой заполнена лишь позиция $8 a$, на самом деле имеет более высокую симметрию, а именно исходную $F d \overline{3} m$. Три максимальные подгруппы $I 4_{1} / a m d, R 3 m$ и $F 4_{1} 32$ соответствуют деформации решетки с изменением сингонии системы, но не приводящие к расщеплению исходной позиции $8 a$. И лишь в одной подгруппе $F \overline{4} 3 m$ происходит расщепление исходной позиции на две: $4 a$ и $4 d$, что позволяет разместить в них атомы разного сорта $\mathrm{Si}$ и С. Эта 
Таблица 1. Симметрия фононов в кристаллах кремния и алмаза (пр. гр. $F d \overline{3} m, \# 227)$

\begin{tabular}{|c|c|c|c|c|c|c|}
\hline Атомы & $\mathbf{q}$ & $\beta$ & $\begin{array}{c}\Gamma \\
(000) \\
m \overline{3} m\end{array}$ & $\begin{array}{c}X \\
(1 / 201 / 2) \\
4 / \mathrm{mmm}\end{array}$ & $\begin{array}{c}L \\
\left(1 / 2 \frac{1 / 2}{3} m\right.\end{array}$ & $\begin{array}{c}W \\
\left(1 / 2 \frac{1}{4} m 23 / 4\right) \\
\overline{4}^{2}\end{array}$ \\
\hline $\mathrm{Si}(\mathrm{C})$ & $\begin{array}{c}2 a \\
(1 / 81 / 81 / 8) \\
(7 / 8 \quad 3 / 83 / 8) \\
\overline{4} 3 m\end{array}$ & $t_{2}(x, y, z)$ & $4^{-}, 5^{+}$ & $1,3,4$ & $1^{+}, 2^{-}, 3^{+}, 3^{-}$ & $1,2,2$ \\
\hline
\end{tabular}

Таблица 2. Симметрия фононов в кубической фазе кристалла карбида кремния (пр. гр. $F \overline{4} 3 m, \# 216$ )

\begin{tabular}{|c|c|c|c|c|c|c|}
\hline Атомы & $\mathbf{q}$ & $\beta$ & $\begin{array}{c}\Gamma \\
(000) \\
\overline{4} 3 m\end{array}$ & $\begin{array}{c}X \\
(1 / 201 / 2) \\
\overline{4} 2 m\end{array}$ & $\begin{array}{c}L \\
(1 / 21 / 21 / 2) \\
3 m\end{array}$ & $\begin{array}{c}W \\
\left(1 / 2 \frac{1 / 4}{4}\right. \\
\end{array}$ \\
\hline $\mathrm{Si}$ & $\begin{array}{c}1 a \\
(000) \\
\overline{4} 3 m\end{array}$ & $t_{2}(x, y, z)$ & 4 & 3,5 & 1,3 & $2,3,4$ \\
\hline $\mathrm{C}$ & $\begin{array}{c}1 d \\
\left(3 / 4 \frac{3}{4} / 43 / 4\right) \\
\overline{4} 3 m\end{array}$ & $t_{2}(x, y, z)$ & 4 & 1,5 & 1,3 & $1,2,4$ \\
\hline
\end{tabular}

Таблица 3. Симметрия фононов в тригональной фазе кристалла карбида кремния (пр. гр. $R 3 m, \# 160$ )

\begin{tabular}{|c|c|c|c|c|c|c|}
\hline Атомы & $q$ & $\beta$ & $\begin{array}{c}\Gamma \\
(000) \\
3 m\end{array}$ & $\begin{array}{c}\mathrm{T} \\
(1 / 21 / 21 / 2) \\
3 m\end{array}$ & 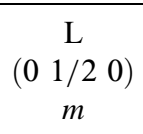 & $\begin{array}{c}\mathrm{F} \\
(1 / 21 / 20) \\
m\end{array}$ \\
\hline $\begin{array}{l}\mathrm{Si} \\
\mathrm{C}\end{array}$ & $\begin{array}{c}3 a \\
(00 z) \\
3 m\end{array}$ & $\begin{array}{c}a(z) \\
e(x, y)\end{array}$ & $\begin{array}{l}1 \\
3\end{array}$ & $\begin{array}{l}1 \\
3\end{array}$ & $\begin{array}{c}1 \\
1,2\end{array}$ & $\begin{array}{c}1 \\
1,2\end{array}$ \\
\hline
\end{tabular}

подгруппа соответствует кубическому карбиду кремния со структурой цинковой обманки. Эта структура допускает дальнейшее понижение симметрии. Группа $F \overline{4} 3 m$ имеет 4 максимальные подгруппы, из которых одна $R 3 m$ соответствует смещению атома углерода из позиции $4 d(3 / 4$ 3/4 3/4) вдоль направления [111]. Двигаясь далее, можно получать различные возможные низкосимметричные структуры, представленные на рис. 1.

Рассмотрим эволюцию фононного спектра при переходе от кубической группы $F d \overline{3} m$ к тригональной $R 3 m$ через промежуточную кубическую $F \overline{4} 3 m$.

Для получения симметрии фононов в точках высокой симметрии зоны Бриллюэна (ЗБ) мы воспользуемся программой SITESYM BCS [11-13]. В табл. 1-3 приведены симметрии фононов в точках высокой симметрии ЗБ в изоструктурных кристаллах кремния и алмаза, в кубической фазе кристалла карбида кремния и в триклинной фазе кристалла $\mathrm{SiC}$ соответственно. В первом столбце табл. 1-3 указаны атомы, расположенные в позициях симметрии (позициях Уайкофа) q, приведенных во втором столбце вместе с их координатами в единицах векторов кристаллографической элементарной ячейки и группой локальной симметрии. В столбцах 4-7 приводятся неприводимые представления (НП) пространственных групп соответствующих кристаллов, описывающие симметрии фононов в точках симметрии ЗБ и индуцированные теми НП $\beta$ локальной группы, по которым преобразуются векторы локальных атомных смещений. Обозначения точек симметрии ЗБ вместе с их координатами в единицах векторов обратной решетки и группами волнового вектора, приведены в заголовках соответствующих столбцов таблиц. Обозначения НП пространственных групп соответствуют работе [22], а обозначения НП локальных групп следуют [23]. Установка пространственной группы и номенклатура позиций Уайкофа выбрана в соответствии с Международными кристаллографическими таблицами [18], но кратность позиций симметрии приводится на одну примитивную ячейку.

Табл. 1-3 позволяют определить, какие фононные моды индуцируются локальными смещениями заданных атомов. Так, например, из табл. 2 видно, что смещения атома $\mathrm{Si}$ преобразующиеся по НП $t_{2}$, индуцируют в точке $\Gamma$ фонон с симметрией $\Gamma_{4}$, в точке $X-$ фононы 
Таблица 4. Правила отбора для рамановских спектров

\begin{tabular}{|c|c|c|c|c|}
\hline Фаза & Симметрия & $\begin{array}{c}\text { Оптические } \\
\text { фононы }\end{array}$ & $\begin{array}{c}\text { Рассеяние } \\
\text { назад }\end{array}$ & $\begin{array}{c}90^{\circ} \text {-геометрия } \\
\text { рассеяния }\end{array}$ \\
\hline $\begin{array}{l}\text { Исходная } \\
\text { кубическая } \mathrm{Si}\end{array}$ & $\begin{array}{l}F d \overline{3} m \\
\# 227\end{array}$ & $T_{2 g}$ & $\begin{array}{l}-x(y z) x \\
-y(x z) y \\
-z(x y) z\end{array}$ & $\begin{array}{l}x(y z) y \\
y(x y) x \\
y(x y) z \\
y(x z) x \\
z(x y) x \\
z(x z) x \\
z(x z) y \\
z(y z) x \\
z(y z) y\end{array}$ \\
\hline \multirow[t]{3}{*}{$\begin{array}{l}\text { Промежуточная } \\
\text { кубическая } \mathrm{SiC}\end{array}$} & \multirow[t]{3}{*}{$\begin{array}{l}F \overline{4} 3 m \\
\# 216\end{array}$} & $T_{2}(\mathrm{LO})$ & $\begin{array}{l}-x(y z) x \\
-y(x z) y \\
-z(x y) z\end{array}$ & - \\
\hline & & $T_{2}(\mathrm{TO})$ & - & $\begin{array}{l}y(x y) x \\
z(x z) x \\
z(y z) y\end{array}$ \\
\hline & & $T_{2}(\mathrm{LO}+\mathrm{TO})$ & - & $\begin{array}{l}x(y z) y \\
y(x y) z \\
y(x z) x \\
z(x y) x \\
z(x z) y \\
z(y z) x\end{array}$ \\
\hline \multirow[t]{6}{*}{ Тригональная SiC } & \multirow[t]{6}{*}{$\begin{array}{l}R 3 m \\
\# 160\end{array}$} & $A_{1}(\mathrm{LO})$ & $\begin{array}{l}-z(x x) z \\
-z(y y) z\end{array}$ & - \\
\hline & & $A_{1}(\mathrm{TO})$ & $\begin{array}{l}-x(y y) x \\
-x(z z) x \\
-y(x x) y \\
-y(z z) y\end{array}$ & $\begin{array}{l}x(z z) y \\
y(z z) x\end{array}$ \\
\hline & & $E(\mathrm{LO})$ & $-y(x x) y$ & - \\
\hline & & $E(\mathrm{TO})$ & $\begin{array}{l}-x(y y) x \\
-x(y z) x \\
-y(x z) y \\
-z(x x) z \\
-z(x y) z \\
-z(y y) z\end{array}$ & $\begin{array}{l}x(y y) z \\
y(x y) z \\
z(x z) y \\
z(y y) x \\
z(y z) x\end{array}$ \\
\hline & & $A_{1}(\mathrm{LO}+\mathrm{TO})$ & - & $\begin{array}{l}x(y y) \mathrm{z} \\
y(x x) z \\
z(x x) y \\
z(y y) x\end{array}$ \\
\hline & & $E(\mathrm{LO}+\mathrm{TO})$ & - & $\begin{array}{l}x(y z) \mathrm{y} \\
y(x x) z \\
y(x y) x \\
y(x z) x \\
z(x x) y \\
z(x y) x \\
z(x z) x \\
z(y z) y\end{array}$ \\
\hline
\end{tabular}


$X_{3}+X_{5}$, в точке $L-$ фононы $L_{1}+L_{3}$, а в точке $W-$ фононы $W_{2}+W_{3}+W_{4}$.

Таким образом, суммируя вклады, даваемые смещениями различных атомов в примитивной ячейке, можно получить колебательное представление в любой точке ЗБ. В кристаллах кремния и алмаза в точке Г оно имеет вид

$$
\Gamma=\Gamma_{4}^{-}+\Gamma_{5}^{+}=T_{1 u}+T_{2 g},
$$

где

$$
\begin{gathered}
\Gamma_{\mathrm{ac}}=\Gamma_{4}^{-}=T_{1 u}, \\
\Gamma_{\mathrm{opt}}=\Gamma_{5}^{+}=T_{2 g} .
\end{gathered}
$$

При замещении одного из атомов кремния в примитивной ячейке на углерод группа симметрии кристалла понижается до группы симметрии цинковой обманки. Симметрия фононов в этом кристалле представлена в табл. 2. В кристалле исчезает центр инверсии и оптическая и акустическая моды $T_{2}$ становятся активными как в спектрах ИК поглощения, так и в рамановских спектрах. В этом кристалле колебательное представление в центре ЗБ имеет вид

$$
\Gamma=2 \Gamma_{4}=2 T_{2},
$$

где

$$
\begin{gathered}
\Gamma_{\mathrm{ac}}=\Gamma_{4}=T_{2}, \\
\Gamma_{\mathrm{opt}}=\Gamma_{4}=T_{2} .
\end{gathered}
$$

При этом для акустической и оптической ветвей наблюдается LO-TO расщепление.

При смещении атома углерода вдоль направления [111] в кубической фазе $\mathrm{SiC}$ симметрия кристалла $F \overline{4} 3 m$ понижается до тригональной $R 3 m$. В этом кристалле колебательное представление в центре ЗБ имеет вид

$$
\Gamma=2 \Gamma_{1}+2 \Gamma_{3}=2 A_{1}+2 E,
$$

где

$$
\begin{gathered}
\Gamma_{\mathrm{ac}}=\Gamma_{1}+\Gamma_{3}=A_{1}+E, \\
\Gamma_{\mathrm{opt}}=\Gamma_{1}+\Gamma_{3}=A_{1}+E .
\end{gathered}
$$

Следовательно, мода $T_{2}$ кубической фазы в тригональной фазе расщепляется на $A_{1}+E$. Величина расщепления пропорциональна величине смещения атома углерода относительно положения (3/4 3/4 3/4). Для этих мод наблюдается также LO-TO расщепление.

Таким образом, эволюция фононных мод в центре зоны Бриллюэна при переходе от исходной кубической к тригональной фазе следующая:

$$
T_{2 g, u} \rightarrow T_{2} \rightarrow A_{1}+E
$$

Аналогичные соотношения для других точек зоны Бриллюэна можно получить, используя программу

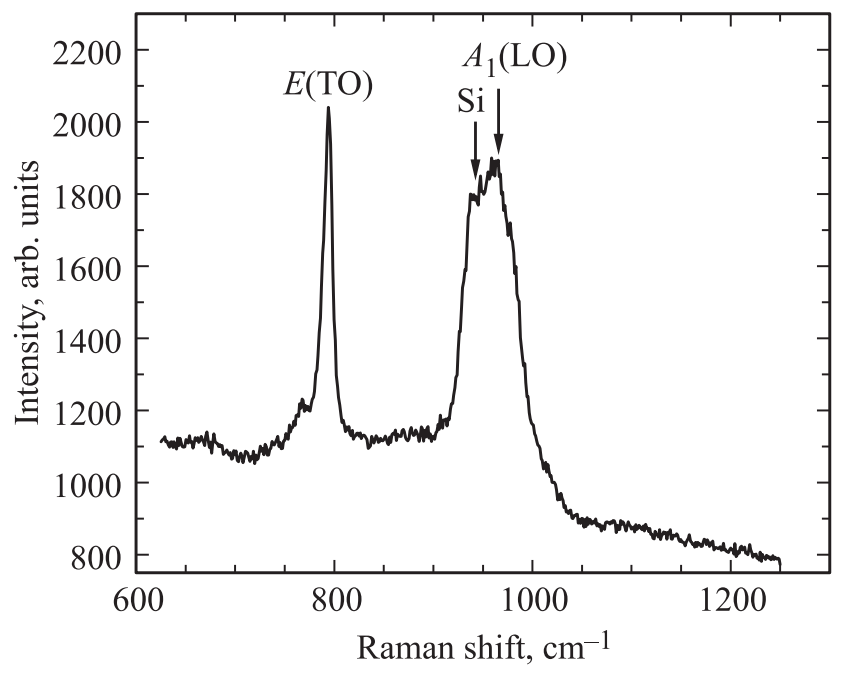

Рис. 2. Рамановские спектры $\mathrm{SiC}$ В спектрах наблюдаются две оптические моды: $A_{1}(\mathrm{LO})$ с частотой $980 \mathrm{~cm}^{-1}$ и $E(\mathrm{TO})$ с частотой $798 \mathrm{~cm}^{-1}$. Линия с частотой $950 \mathrm{~cm}^{-1}$ соответствует кремниевой подложке.

CORREL [11]. Например, при переходе из исходной кубической фазы в промежуточную кубическую фононные моды в точке $L$ преобразуются следующим образом:

$$
\begin{aligned}
& L_{1}^{+} \rightarrow L_{1}, L_{2}^{-} \rightarrow L_{1}, \\
& L_{3}^{+} \rightarrow L_{3}, L_{2}^{-} \rightarrow L_{3} .
\end{aligned}
$$

и далее при переходе в тригональную фазу

$$
L_{1} \rightarrow T_{1}+L_{1}, \quad L_{3} \rightarrow T_{3}+L_{1}+L_{2} .
$$

Правила отбора для различных геометрий рассеяния получим, используя программу POLARIZATION SELECTION RULES [11-13]. Результаты анализа приведены в табл. 4. Для кубических фаз оси $x, y, z$ направлены по ребрам куба, а в тригональной фазе ось $z$ направлена вдоль оси третьего порядка по пространственной диагонали [111] исходной кубической решетки.

На рис. 2 представлены неполяризованные рамановские спектры в геометрии назад при направлении падающего и рассеянного света вдоль оси $z$. В этом случае согласно правилам отбора (табл. 4) в спектрах должны наблюдаться две оптические моды: $A_{1}(\mathrm{LO})$ и $E(\mathrm{TO})$. Именно эти линии с частотами 980 и $798 \mathrm{~cm}^{-1}$ соответственно и наблюдаются в нашем эксперименте.

Таким образом, в настоящей работе было получены все возможные структурные типы, которые можно получить из исходной структуры кремния, пошагово понижая ее симметрию либо добавлением новых атомов, либо смещениями атомов в элементарной ячейке. Определена трансформация фононного спектра при переходе от исходной структуры кремния к тригональной структуре карбида кремния через кубическую фазу $\mathrm{SiC}$. Полученные правила отбора для рамановских спектров позволили идентифицировать линии, наблюдавшиеся в эксперименте. 


\section{Список литературы}

[1] В.Г. Дубровский. Теория формирования эпитаксиальных наноструктур. Физматлит, М. (2009). 351 с.

[2] O.P. Pchelyakova, Yu.B. Bolkhovityanova, A.V. Dvurechenskiia, A.I. Nikiforova, A.I. Yakimova, B. Voigtlände. Thin Solid Films 367, 75 (2000).

[3] Р. Куэй. Электроника на основе нитрида галлия. Техноcфepa. M. (2011). 592 c.

[4] B.H. Bairamov, R.A. Evarestov, Yu.E. Kitaev, E. Jahne, M. Delaney, T.A. Gant, M.V. Klein, D. Levi, J. Klem, H. Morkoc. J. Phys. Chem. 95, 10772 (1991).

[5] Yu.E. Kitaev, A.G. Panfilov, P. Tronc, R.A. Evarestov. J. Phys.: Condens. Matter 9, 257 (1997).

[6] Yu.E. Kitaev, A.G. Panfilov, P. Tronc, R.A. Evarestov. J. Phys.: Condens. Matter 9, 277 (1997).

[7] Yu.E. Kitaev, M.F. Limonov, P. Tronc, G.N. Yushin. Phys. Rev. B 57 (22), 14209 (1998).

[8] V.Yu. Davydov, E.M. Roginskii, A.N. Smirnov, Yu.E. Kitaev, M.A. Yagovkina, R.N. Kyutt, M. Rozhavskaya, E.E. Zavarin, W.V. Lundin, M.B. Smirnov. Phys. Status Solidi A 210, 484 (2013).

[9] M.I. Alonso, M. Cardona, G. Kanellis. Solid State Commun. 69, 479 (1989).

[10] P. Molinas i Mata, M.I. Alonso, M. Cardona. Solid State Commun. 74, 347 (1990).

[11] M.I. Aroyo, A. Kirov, C. Capillas, J.M. Perez-Mato, A. Wondratschek. Acta Cryst. Section A: Found. Crystallogr. 62, 115 (2006); www. cryst.ehu.es

[12] M.I. Aroyo, J.M. Perez-Mato, C. Capillas, E. Kroumova, S. Ivantchev, G. Madariaga, A. Kirov, A. Wondratschek. Z. Kristallogr. 221, 15 (2006).

[13] M.I. Aroyo, J.M. Perez-Mato, D. Orobengoa, E. Tasci, G. de la Flor, A. Kirov. Bulg. Chem. Commun. 43, 183 (2011).

[14] S.A. Kukushkin, A.V. Osipov. J. Phys. D 47, 313001 (2014).

[15] С.А. Кукушкин, А.В. Осипов, Н.А. Феоктистов. ФТТ 56, 1457 (2014).

[16] С.А. Кукушкин, А.В. Осипов. ФТТ 56, 761 (2014).

[17] Yu.E. Kitaev, P. Tronc. ФTT 54, 490 (2012).

[18] International Tables for Crystallography. V.A: Space Group Symmetry / Ed. T. Hahn. Kluwer Academic Publishers, Dordrect (2002).

[19] H. Bärnighausen. Math. Chem. 9, 139 (1980).

[20] E. Koch. Acta cryst. A 40, 593 (1984).

[21] A. Fadda, G. Fadda. Phys. Rev. B 82, 104105 (2010).

[22] A.P. Cracknell, B.L. Davies, S.C. Miller, W.F. Love. Kronecker Product Tables. V. 1. General Introduction and Tables of Irreducible Representations of Space Groups. IFI / Plenum, N. Y. (1979).

[23] C.J. Bradley, A.P. Cracknell. The Mathematical Theory of Symmetry in Solids. Clarendon, Oxford (1972). 